\title{
CARACTERIZACIÓN Y ACTUALIZACIÓN DEL MODELO PEDAGÓGICO DEL PROGRAMA DE DERECHO DE LA UNIVERSIDAD SURCOLOMBIANA
}

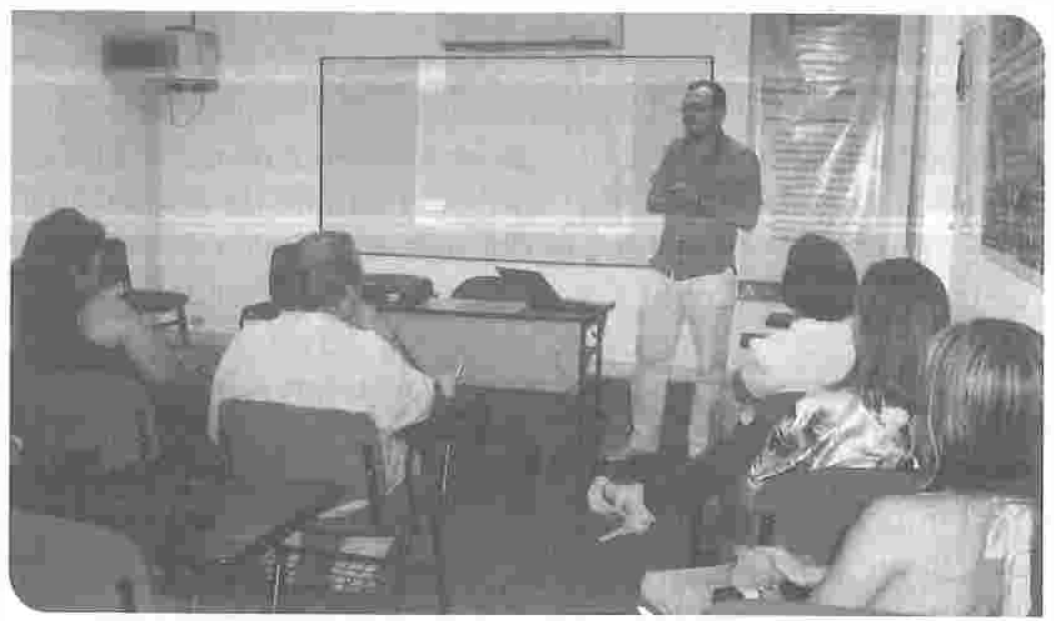

OSCAR HUBER ZÚÑIGA CÓRDOBA ${ }^{45}$

\section{PLANTEAMIENTO DE LA PREGUNTA O PROBLEMA DE INVESTIGACIÓN Y SU JUSTIFICACIÓN}

La carencia de fundamentos y de una doctrina curricular que irradie una verdadera reforma, su intervención adrede sin una técnica adecuada, genera profundas preocupaciones, pues cada profesional del derecho propone una intervención del currículo atendiendo a la preferencia de un área en específico, dándole prioridad a la misma, perdiendo de vista factores trascendentales que son resultado de la investigación y la indagación permanente.

En palabras de LÓPEZ JIMÉNEZ y PUENTES DE VELÁSQUEZ, en el libro Modernización Curricular de la Universidad Surcolombiana, una experiencia de Integración e Interdisciplinariedad, como se identificó en la referenciada investigación, se pudo advertir en el Programa de Derecho la hegemonía por la insularidad, la fragmentación, la clasificación y enmarcación de saberes fuertemente delimitados y jerarquizados. 
En este orden de ideas, en enhor abuena se realizó la presente investigación, la cual se armoniza con la propuesta del Grupo de Investigaciones PACA (Programa de Acción Curricular Alternativo), Categoria A de Colciencias, tendiente a consolidar un modelo de formación alternativo a la realidad educativa actual, fundamentado en la construcción y consolidación de un Modelo Pedagógico integrado, soportado en procesos asociados a la integralidad, la transversalidad, la interdisciplinariedad y la transdisciplinariedad. Se realizó un análisis y una revisión evaluativa de la estructura cur ricular del Programa de Derecho de la Universidad Surcolombiana, dentro de un ambiente de discusión, análisis y reflexión crítica, con el Comité de Currículo, el Comité de Autoevaluación, el Consejo de Facultad de Derecho y el Consejo Académico de la Universidad, dentro de una acción coherente, rigurosa, consistente, completa, democrática, moderna y pertinente del proceso de formación del programa de derecho de la Usco, cuyos resultados, incluso, lograron ser insumos esenciales en la obtención de la Acreditación de Calidad del Programa,

Pero lo más importante, es haber logrado una dinámica que se identificó con la propuesta, que la interiorizara y la asumiera como suya ${ }_{r}$ conformando un estilo de trabajo que le aposto al objetivo de la investigación, que convergió con la necesidad de la autoevaluación y que finalmente logró consolidar una verdadera cultura de la autoevaluación y de la optimización en la prestación del servicio educativo. Dentro de la Propuesta Alternativa lider ada por PACA, además de continuar con las banderas de la Modernización Curricular de la Universidad Surcolombiana, fue el propósito central de esta investigación, lograr una verdadera transformación de la estructura curricular del Programa de Derecho, a partir del formas y procesos de integración e interdisciplinariedad curricular; igualmente se busca que sirva de sustento y referencia para los demás programas de la Universidad, pero de la misma forma, se espera encontrar resonancia en las escuelas de derecho del pais, pues ciertamente consider amos que de la forma de concebir y aplicar el derecho, depende en gran medida, la legitimidad del propio Estado.

En consecuencia se plantearon las siguientes interrogantes:

Preguntas directrices, guías u orientadoras:

¿Cómo identificar y evaluar el Modelo Pedagógico del Programa de Derecho de la Universidad Surcolombiana?

¿Qué elementos debe contemplar el modelo pedagógico, el curriculo, y el plan de estudios del programa de derecho de la Universidad Surcolombiana para su actualización? 
¿Cómo lograr incorporar estrategias curriculares innovadoras de interacción pedagógica dentro del proceso de formación que garantice la integración y la interdisciplinariedad, a partir de la formulación de un modelo pedagógico?

Pregunta Central de la Investigación:

¿Cuál puede ser el Modelo Pedagógico renovador del proceso de formación profesional de los estudiantes del Programa de Derecho de la Universidad Surcolombiana?

\section{JUSTIFICACIÓN}

El Programa de Derecho de la Universidad Surcolombiana plantea y adopta como Modelo Pedagógico el enfoque crítico, que para el cumplimiento de su Misión y el logro de su Visión, busca la formación de profesionales comprometidos con los problemas de orden social, con un alto grado de sensibilidad, asumiendo posiciones que se identifican con la reivindicación de la democracia y el respeto por la dignidad humana. Se propone la transformación de la sociedad para la igualdad social. Igualmente, apoyado en la investigación-acción- participación, interviniendo a la comunidad a través de proyectos sociales y culturales.

A su turno, el modelo pedagógico se alimenta del enfoque constructivista, teniendo como propósito la construcción propia del conocimiento a través del aprender haciendo, aprender a aprender, aprender a investigar, aprender a convivir con otros y con la naturaleza.

Pese a que efectivamente existen documentos que expresan al menos formalmente un Modelo Pedagógico, el mismo no ha sido el resultado de un proceso de investigación y menos ha nacido de las entrañas del programa, de tal forma que resulta interesante indagar sobre su conocimiento y aplicación, esto es sobre su validez, pero lo más importante, su legitimidad, de si existe identidad entre lo que quiere y lo que se ha establecido por el Programa, y lo más importante, si es pertinente.

Como se ha venido planteando, el Programa ha padecido serios inconvenientes académicos y administrativos desde su nacimiento. Se han realizado reformas curriculares sin el lleno de los requisitos estatutarios y sin la utilización adecuada de criterios pedagógicos y metodológicos pertinentes, lo que ha generado situaciones caóticas principalmente en el estudiantado por el tránsito de un plan de estudio a otro y otro de forma desarticulada. Pero también debe manifestarse claramente que no todo es negativo en el Programa, como puede verse de la lectura de los resultados de las pruebas Saber Pro. Por ello 
resulta atractivo indagar sobre el modelo pedagógico institucionalizado, así como del modelo oculto, el que efectivamente se desenvuelve en las aulas del programa.

Es aquí en donde se abre la oportunidad de incluso formular un modelo pedagógico propio, que nazca desde las bases del propio programa, que se identifique con sus particularidades y que manifieste su sentido teleológico, su ethos, su razón de ser y los valores que lo inspiran y justifican. Es por ello que el objeto de la investigación se orientó hacia, la definición, si es necesario o no, de un nuevo modelo pedagógico, construido por docentes, estudiantes, administrativos, egresados y empleadores, al interior del comité de currículo del programa, que consulte con los mismos y con el trabajo realizado desde la base, esto es, desde el mismo espacio del aula y que es materialmente en donde se construye el conocimiento.

El Proyecto Educativo del Programa de Derecho señala que para el cumplimiento de su Misión, y el logro de su Visión, la Universidad Surcolombiana en general, plantea y construye el Modelo Pedagógico Integrado e interdisciplinario, cuyas políticas son desarrolladas y asesoradas por el Comité Central de Currículo, para lo cual, conforme a los principios y fundamentos del Proyecto Educativo Institucional, se acogen los referentes de la Escuela Crítica y el Constructivismo Pedagógico. Dice igualmente, que siguiendo las características del "Modelo Pedagógico integrado" que enfatiza las relaciones entre las metas, las prácticas pedagógicas, los contenidos, los criterios de evaluación y el desarrollo humano, el Programa de Derecho propugna por la implementación de redes de integración y transversalidad de ejes entre contenidos, áreas y componentes, mediante: la Investigación, la práctica del consultorio jurídico y concepción didáctica.

Empero, durante la transición del plan tradicional por asignaturas al expresado en créditos, se incurrió en fehacientes falencias curriculares y pedagógicas, en donde primó la insularidad, la atomización y la jerarquización de las áreas y del conocimiento del derecho. Esto además llevo a que se afectará la situación académica de los estudiantes, ante los cambios intempestivos y abruptos en la malla curricular.

\section{LOS OBJETIVOS}

\section{GENERAL}

Formular un Modelo Pedagógico, dentro de un proceso de actualización y modernización curricular a partir de una propuesta alternativa, construida desde sus particularidades y necesidades propias, definiendo estrategias de integra- 
ción, de interdisciplinariedad, de transdisciplinariedad y complementariedad dentro del proceso aprendizaje para el Programa de Derecho de la Universidad Surcolombiana.

\section{ESPECÍFICOS}

Realizar la caracterización y evaluación tanto del Modelo Pedagógico, como del Currículo y el Plan de Estudios del Programa de Derecho de la Universidad Surcolombiana.

Redefinir la teleología y la fundamentación pedagógica del Programa de Derecho (la didáctica, la evaluación, la formación por competencias, el plan de estudio, los propósitos de formación, ob jetivos, entre otros).

Diseñar una propuesta curricular para la actualización de la escuela de derecho, desde una perspectiva inter disciplinaria, transdisciplinaria, flexible y mediante la formulación de núcleos problemáticos.

\section{METODOLOGÍA PROPUESTA}

La investigación desarrollada se enmarca dentro de las Ciencias Sociales, la cual adopta un enfoque cualitativo, basado en la complementariedad a partir de la integración de acciones relacionadas con la revisión teórica y conceptual, el análisis de documentos oficiales e institucionales, la caracterización del objeto de estudio, interrelacionando así mismo a los actores o informantes, atendiendo igualmente la consulta de los participantes involucrados, con el objeto de comprender en detalle las estructuras que materialmente se desenvuelven en las relaciones curriculares y pedagógicas del Programa de Derecho.

\section{PROCESO METODOLÓGICO:}

Enfoque Epistemológico: Teniendo en cuenta la problemática a abordar, se considera que el enfoque epistemológico más conveniente para desarrollar la presente investigación es el de carácter analítico- descriptivo, puesto que comprenderá la descripción, registro, análisis e interpretación de teorías y conceptos, la revisión y valoración de documentos, así mimo comprende el análisis de las realidades sociales propias de la acción pedagógica y didáctica del programa.

Análisis documental: Se realizó una revisión documental del Programa de Pregrado de Derecho que ofrece la Universidad Surcolombiana, con el fin de evaluar y caracterizar el Modelo Pedagógico del Programa de Derecho, así como los aspectos propios de la didáctica, la evaluación, las competencias, la 
relación educador educandos, plan de estudio, Proyecto Educativo, procesos açadémicos, perfil profesional, misión, visión, propósitos de formación, objetivos, entre otros.

Discusión, análisis y construcción de consensos: En la Investigación se realizaron principalmente reuniones de discusión, análisis y replanteamientos curriculares en los diferentes grupos e instancias académicas del Programa de Derecho de la Universidad Surcolombiana y de otras universidades del país.

Se desarrolló un enfoque investigativo basado en la complementariedad, a partir de la integración de acciones relacionadas con la revisión teórica y conceptual, el análisis de documentos oficiales e institucionales, la caracterización del objeto de estudio y el diseño de una propuesta curricular.

Población y Muestra: Incluye distintos estamentos de la comunidad universitaria:
a- Directivos
b- Docentes
c- Estudiantes

Dentro de este punto, es de resaltar que a partir de la discusión, el debate, la reflexión, el análisis, la confrontación y la crítica, se estructuró paso a paso, cada uno de los insumos y elementos que conforman la nueva propuesta de Modelo Pedagógico.

\section{REFERENTE TEÓRICO}

Inicialmente es de destacar que muy poco, porno decir nada, se ha escrito en materia de Modelos Pedagógicos en Derecho, limitándose la literatura encontrada a la enseñanza del derecho, pero no exactamente a un modelo estructurado de currículo y de modelo pedagógico.

\section{a) La Constitución Política de 1991 y el Estado Social de Derecho.}

Sin lugar a equívocos, la promulgación de la Constitución Política de 1991 y la vigencia de la fórmula política del Estado Social de Derecho, a la luz del Artículo primero de la Constitución, simboliza un cisma y un hito en el constitucionalismo yen la concepción del derecho en Colombia. Como consecuencia de la manifestación del inconformismo popular de diferentes sectores frente al antiguo sistema constitucional, los acuerdos de paz celebrados con el M-19, y acontecimientos violentos como la campaña de exterminio de la Unión Patriótica y los asesinatos de varios candidatos presidenciales, se expide una nueva Constitución política. 
Se daba así un paso, después de varios intentos frustrados de reforma, a un cambio constitucional que contó con la participación de distintos representantes de la sociedad elegidos popularmente, y que superaba las difíciles circunstancias políticas y los formalismos jurídicos de la Constitución de 1886. Jaime Buenahora describe este proceso de la siguiente forma:

"No sólo estábamos ante la primera Asamblea Nacional Constituyente de la historia colombiana elegida popular y democráticamente, sino ante una Asamblea que era expresión real de toda la Nación, conformada por representantes y voceros de las distintas regiones, pluralista ideológicamente y equilibrada en la distribución de las fuerzas politicas y sociales."

El papel protagónico del bipartidismo en la Asamblea Nacional Constituyente puede generar una visión menos optimista de este proceso. Sin embargo, es innegable que frente a las constituciones anteriores, la de 1991 representa un logro significativo en materia de participación democrática y pluralismo politico, reflejado en un texto generoso en derechos sociales e individuales, mecanismos de protección de los individuos y defensor del Estado de Derecho.

Este nuevo carácter, alimentado además por la necesidad de generar un poder político más equilibrado y alejado de los vicios del presidencialismo, es un sueño que quedó plasmado en el nuevo texto constitucional en su artículo primero:

"Colombia es un Estado social de derecho, organizado en forma de república unitaria, descentralizada, con autonomia de sus entidades territoriales, democrática, participativa y pluralista, fundada en el respeto de la dignidad humana, en el trabajo y la solidaridad de las personas que integran yen la prevalencia del interés general."

El nuevo término "social", ahora agregado a la clásica fórmula del Estado de Derecho, no debe ser entendido como una simple muletilla retórica que proporciona un elegante toque de filantropía a la idea tradicional del derecho y del Estado (Sentencia T 40ó de 1992 de la Corte Constitucional, Ponente Ciro Angarita). Una larga historia de transformaciones institucionales en las principales democracias constitucionales del mundo, está presente para dar testimonio de la trascendencia de este concepto. Como lo ha señalado la propia Corte Constitucional: la incidencia del Estado Social de Derecho en la organización sociopolítica puede ser descrita esquemáticamente desde dos puntos de vista: cuantitativo y cualitativo. Lo primero suele tratarse bajo el tema del Estado Bienestar (welfare State, stato del benessere, L'Etat Providence) y lo segundo bajo el tema de Estado Constitucional Democrático. La delimitación 
entre ambos conceptos no es tajante; cada uno de ellos hace alusión a un aspecto específico de un mismo asunto. Su complementariedad es evidente.

El Estado Bienestar surgió a principios de siglo en Europa como respuesta a las demandas sociales; el movimiento obrero europeo, las reivindicaciones populares provenientes de las revoluciones Rusa y Mexicana y las innovaciones adoptadas durante la república de Weimar, la época del New Deal en los Estados Unidos, sirvieron para transformar el reducido Estado liberal en un complejo aparato político-administrativo jalonador de toda la dinámica social. Desde este punto de vista el Estado Social puede ser definido como el Estado que garantiza estándares mínimos de salario, alimentación, salud, habitación, educación, asegurados para todos los ciudadanos bajo la idea de derecho y no simplemente de caridad (H.L. Wilensky, 1975).

El Estado constitucional democrático ha sido la respuesta jurídico-política derivada de la actividad intervencionista del Estado. Dicha respuesta está fundada en nuevos valores-derechos consagrados por la segunda y tercera generación de derechos humanos y se manifiesta institucionalmente a través de la creación de mecanismos de democracia participativa, de control politico y jurídico en el ejercicio del poder y sobre todo, a través de la consagración de un catálogo de principios y de derechos fundamentales que inspiran toda la interpretación y el funcionamiento de la organización política.

Estos cambios han producido en el derecho no sólo una transformación cuantitativa debida al aumento de la creación jurídica, sino también un cambio cualitativo, debido al surgimiento de una nueva manera de inter pretar el derecho, cuyo concepto clave puede ser resumido de la siguiente manera: pérdida de la importancia sacramental del texto legal entendido como emanación de la voluntad popular y mayor preocupación por la justicia material y por el logro de soluciones gue consulten la especificidad de los hechos. Estas características adquieren una relevancia especial en el campo del derecho constitucional, debido a la generalidad de sus textos y a la consagración que allí se hace de los principios básicos de la organización política. De aquí la enorme importancia que adquiere el juez constitucional en el Estado Social de Derecho.

La complejidad del sistema, tanto en lo que se refiere a los hechos objeto de la regulación, como a la regulación misma, hace infructuosa la pretensión racionalista que consiste en prever todos los conflictos sociales posibles para luego asignar a cada uno de ellos la solución normativa correspondiente. En el sistema jurídico del Estado Social de Derecho se acentúa de manera dramática el problema -planteado ya por Aristóteles- de la necesidad de adaptar, corregir, acondicionar la aplicación de la norma por medio de la intervención 
del juez. Pero esta intervención no se manifiesta sólo como el mecanismo necesario para solucionar una disfunción, sino también, y sobre todo, como un elemento indispensable para mejorar las condiciones de comunicación entre el derecho y la sociedad, es decir, para favorecer el logro del valor justicia (de la comunicación entre derecho y realidad), así ello conlleve un detrimento de la seguridad jurídica.

Es justamente aquí, en esta relación entre justicia y seguridad jurídica, donde se encuentra el salto cualitativo ya mencionado: El sistema jurídico creado por el Estado liberal tenía su centro de gravedad en el concepto de ley, de código. La norma legal, en consecuencía, tenía una enorme importancia formal y material, como principal referente de la validez y como depositaria de la justicia y de la legitimidad del sistema. En el Estado intervencionista se desvanece buena parte de la importancia formal (validez) por la importancia material (justicia) de la ley.

La nueva Constitución colombiana recoge ampliamente los postulados normativos del Estado Social de Derecho. Ello se comprueba no solo al repasar lo consagrado en la lísta de los principios y de la Carta de derechos, sino también en la organización del aparato estatal. Como se puede observar, una de las características más relevantes del Estado Social de Derecho consiste en la importancia que adquiere el juez en sus relaciones con el legislador y con la administración. Buena parte de ella se deriva del nuevo papel que juegan los principios constitucionales en las decisiones judiciales y su relación con los valores y normas de la Carta.

A partir de este nuevo contexto, se puede concluir que la forma de concebir el derecho no depende ya de una lógica formal. Por el contrario, desde 1991 el objetivo fundamental es impartir justicia con base en decisiones argumentadas, donde se reivindican los derechos fundamentales y la dignidad humana, yes desde este contexto y perspectiva que debe desarrollarse el proceso pedagógico y de enseñanza en derecho.

\section{PROPUESTA CURRICULAR ALTERNATIVA}

Frente a los anteriores resultados, existe un total consenso en la necesidad de reaccionar a través de un proceso de de-construcción, que permita liderar acciones que garanticen la pertenencia institucional, para un desarrollo y replanteamiento curricular del Programa de Derecho, en donde se pueda construir un nuevo proyecto que reivindique materialmente lo interdisciplinar, lo transdisciplinar y lo trasversal, como prenda de garantía de una formación integral, dentro de una renovación de su Proyecto Educativo. 
Se trata de integrar lo administrativo, lo axiológico, lo académico, lo curricular, lo investigativo, con lo institucional, pero también con otras agencias tales como la familia, el sector productivo, lo político, entro otros, dando paso a una forma alternativa de organización soportada en la integración y la interdisciplinariedad, para transformar y de-construir sus estructuras, basado en la formación por competencias, brindando pertinencia e identidad al proceso de formación del profesional del Programa de Derecho de la Universidad Surcolombiana.

Con este propósito, mediante la presente investigación se formula un Proyecto Educativo Renovador, el cual interviene la realidad encontrada en aspectos sustantivos como:

\section{Fundamentación Teórica y Metodológica Del Programa}

El modelo pedagógico es el contexto, la representación de las relaciones, sus herramientas conceptuales, la forma de desarrollar los procesos académicos, el derrotero filosófico y la impronta de formación en los programas y/o escuelas, con el objeto de definir los fines (para qué-propósitos de formación); la concepción de desarrollo del estudiante (quién-competencias); los contenidos (qué), la estructura curricular, los componentes flexibles básicos y complementarios, las áreas y los proyectos transversales; la metodología (cómo-actividades presenciales e independientes), los procesos de investigación, de formación, de prácticas, modelos de evaluación y promoción; ambientes de aprendizaje (dónde-escenarios) salones de clase, bibliotecas, laboratorios, sitios de práctica. Por tanto, es el instrumento analítico para describir, organizar e inteligir la multiplicidad, la diversidad, las contingencias y estructuras que presenta una corriente pedagógica.

\section{Concepción pedagógica - Modelo pedagógico}

El Programa de Derecho mediante la presente investigación, logró conformar un grupo de docentes, estudiantes, administrativos y egresados adelantó, quienes dentro de un proceso de indagación, realizaron un debate acerca del Modelo Pedagógico, dentro de una discusión participativa y ampliada, con el propósito de abordar su pertinencia y vigencia, su aplicación por parte de los docentes del Programa, así como su conocimiento y manejo por toda la comunidad educativa. Teniendo igualmente en cuenta, las dinámicas históricas y las reales condiciones actuales en la formación de los profesionales del derecho.

Así pues el equipo advirtió que aun cuando el modelo no goza de una aplicabilidad total por parte de algunos docentes catedráticos (en cierta medida por tradicionalismo arraigado en los métodos de enseñanza), es también cierto y verificable que los procesos académicos desarrollados al interior del Programa 
se adelantan de manera satisfactoria, producto de lo cual se han consolidado resultados académicos importantes en las pruebas ECAES, hoy Saber Pro, en las que el Programa ha obtenido niveles destacables, en un mecanismo que es aceptado por la comunidad académica como criterio de comprobación del logro de las competencias alcanzadas por el estudiante, pero de igual manera resulta relevante el impulso que el mismo modelo ofrece al desarrollo y consolidación de la actividad investigativa y de proyección social.

Como resultado del proceso surtido se concluyó, que la respuesta necesaria para el Programa de Derecho debería ser la adopción de un Modelo Pedagógico de Indagación Sistemática, estructurado en Núcleos Temáticos Problemáticos, caracterizado par la Disciplinariedad, la Interdisciplinariedad, la Trasdisciplinariedad y la Complementariedad, desde perspectiva alternativa y neosistémica, tras la adopción de estrategias que involucren un proceso de divulgación efectivo entre el cuerpo docente y estudiantil, que permita trascender lo meramente explicativo para centrarse en el logro de una verdadera asimilación por parte de los estamentos, especialmente el de maestros.

Portal razón el Programa de Derecho de la Universidad Surcolombiana plantea y adopta un Modelo Pedagógico que conceptualmente se alimenta de las teorías del enfoque crítico, desde una perspectiva alternativa, que como garantía del cumplimiento de su Misión y el logro de su Visión, propende por la formación de profesionales comprometidos con los problemas de orden social, con un alto grado de sensibilidad, con la asunción de posiciones que se identifiquen con la reivindicación de la democracia y el respeto por la dignidad humana, en apoyo de elementos radicados en otras escuelas o corrientes pedagógicas, que permitan proponer la transformación de la sociedad para la igualdad social.

Bajo esta línea de razonamiento, se parte de la premisa que dicho enfoque se ocupa de los problemas del orden social, asume posiciones politicas y emancipadoras, se identifica con el desarrollo de la sociedad, la primacía del interés general, la transformación de la sociedad en procura de lograr una igualdad material, al tiempo que privilegia las formas de trabajos politécnicos y polivalentes ligados a la producción. Actualmente se ubica en los enfoques de la pedagogía crítica y la educación popular, se apoya en la investigación-acción-participación, la teoría crítica de la sociedad, la acción comunicativa y la emancipación, donde sus principales representantes son Makarenko, Freire, Freinet, Magendzo y Vigostky. En lo relacionado con el Contexto, es necesario avanzar en un proceso de detección de necesidades que permitan escrutar de una manera compartida él, o, los problemas, que van a dar lugar a la formulación del propósito y el perfil de formación del programa acadệmico. 
Mediante la adopción de este modelo, se pretende replantear la relación educador-educando, a partir de una doctrina pedagógica que propicie los principios democráticos y participativos; asuma una estrategia metodológica renovadora del proceso de formación profesional de los estudiantes del Programa de Derecho con énfasis en el Derecho como fenómeno social, e instrumento de cambio, donde el abogado debe ser un forjador de cambio social.

Se formula una formación o proceso de aprendizaje por competencias, en donde se aspira a la formación de profesionales integrales, de ciudadanos civilizados, respetuosos del estado social de derecho y portavoces de una nueva cultura del respeto por los derechos humanos. No obstante, no es incompatible esta propuesta con la orientación de determinados contenidos básicos disciplinares, por medio de la exposición o clase magistral.

Éste último aspecto, se soporta en el compromiso con la sociedad y el Estado, en desarrollo del cometido de la función social que demanda la profesión de abogado, como garantía de una adecuada y optima defensa técnica, y del deber de colaboración con una recta y cumplida administración de justicia, de tal forma que los estudiantes deben estar irrestrictamente capacitados para un optimo desempeño en cualquiera de los escenarios de su profesión. Por tanto, se reconoce la necesidad imperiosa de una sólida fundamentación teórica y práctica, para un adecuado desempaño de la profesión del Abogado, reconociendo que existen conceptos y contenidos que ineludiblemente deben ser asimilados por los educandos, por constituir principios que gobiernan las actuaciones e instalamentos para ejercer correctamente la profesión en procura de una efectiva tutela de los derechos de las personas.

\section{Estrategia Curricular Interdisciplinaria: Núcleos Temáticos Problemáticos.}

Un Modelo Alternativo de Acción Curricular implica la construcción de estrategias curriculares que consulten la naturaleza alternativa del proceso de construcción de una nueva cultura académica, que permíta construir nuevas identidades profesionales y pedagógicas. Por ello se proponen los Núcleos Temáticos Problemáticos como propuesta curricular interdisciplinaria, entendida como el conjunto de conocimientos afines que posibilitan definir prácticas y procesos de investigación en torno a un objeto/problema y basado en el desarrollo de competencias. Esto implica la construcción de estrategias que garanticen la relación teoría-práctica y la construcción de acciones participativas entre individuos y grupos en la diversidad de soluciones propuestas. Se considera una estrategia que permite integrar un campo de problemas con un campo de conocimientos propios de la formación de un profesional. 
Trabajar con la estrategia Núcleo Temático y Problemático, advierte una correlación directa con el propósito de formación que orienta y determina el desarrollo académico del proceso formativo, en la medida en que se convierte en un dispositivo que facilita la integración de la formación, la investigación y la proyección social, considerados procesos determinantes en la identidad de las instituciones del nivel de la educación superior. La implementación de un Modelo Pedagógico Integrado, de Indagación Sistemática, fundamentado en Núcleos Temáticos Problemáticos, demandó del diseño de diversas estrategias didácticas y la implementación de una cultura para el trabajo autónomo, por lo que se desarrollará un plan de mejoramiento que permita transformar la mentalidad del docente frente a la dinámica de enseñanza - aprendiza je, así como alcanzar una flexibilización en los aspectos pedagógicos y administrativos, buscando depurar los contenidos curriculares, lo que permitirá organizar una tendencia de valoración de los créditos hasta lograr una relación más estandarizada.

Además de lo anterior, existen otras actividades académicas que apunta al logro de una formación integral y flexible por competencias, como los son las practicas extramuros, en las que se desarrollar acciones extracurriculares que buscan un mayor acercamiento del estudiante al proceso educativo, gracias al aprendizaje basado en situaciones reales, promoviendo al mismo tiempo mayor grado posible de articulación entre las exigencias del mundo productivo y la formación profesional para el desarrollo humano.

Igualmente hace parte integral del proceso de formación las actividades desarrolladas en investigación, a través de los grupos y semilleros, así como las actividades de proyección social remunerada y asistencia; todas éstas, permiten un desarrollo integral y una cultura por la búsqueda constante del conocimiento, a fin de ponerlo al servicio de la comunidad.

\section{Formación por Competencias}

El Propósito Fundamental del Programa es formar profesionales comprometidos con la defensa y promoción de los derechos fundamentales, el respeto de la dignidad humana ${ }_{f}$ el pluralismo, la libertad de pensamiento, la solidaridad, la igualdad material, la construcción de un orden justo, la convivencia pacifica y la reivindicación y defensa del Estado Social de Derecho.

\section{Formación por competencias - Concepción de desarrollo en el estudiante}

El Programa ha establecido como prioritarias las siguientes competencias, necesarias dentro del proceso de formación profesional y humana en el ámbito 
jurídico, las cuales se ajustan a las particularidades propias del Programa, a las consideraciones del ICFES para la elaboración de los términos de las pruebas Saber-Pro en Derecho, a las exigencias de la Resolución 2768 de 2003, al igual que a los parámetros adoptados por la Universidad:

Competencia cognitiva disciplinar: entendida como la capacidad de conocer, describir, experimentar, transformar y producir conocimjentos jurídicos y socio-jurídicos fundamentales.

Competencia de administración del conflicto: con ella se busca la capacidad para prevenir, identificar, proponer soluciones, hacer control y seguimiento del conflicto e intervenir en él mediante un manejo integral del mismo.

Competencia comunicativa: se refiere a la capacidad para comprender y utilizar de forma precisa el lenguaje jurídico, de tal manera que pueda comunicar sus ideas con claridad y coherencia en todos los escenarios del ejercicio de su profesión. La competencia de comunicación engloba las funciones de comprender, escuchar, hablar, leery escribir, junto al lenguaje gestual (sistemas simbólicos).

Competencia de investigación juridica: es la capacidad de recopilar, sistematizar, analizar, valorar y tratar situaciones, condiciones y posibilidades de la realidad socio-jurídica, en función de la generación de conocimiento científico.

Competencia de la responsabilidad profesional: comprende uno de los principales retos de formación al interior del programa, al propender por la actuación profesional y humana con honestidad, diligencia, transparencia y responsabilidad en la asesoría, representación y defensa de los intereses de las personas que salvaguarda y defiende en sede judicial o extrajudicial.

Abogado de audiencia: de cara a las nuevas dinámicas procesales del derecho en Colombia, en donde la oralidad cobra un papel protagónico en la actualidad yen un futuro inmediato, el Programa dentro de su proceso de modernización establece el desarrollo de técnicas de juicio oral, la oralidad, la oratoria, la persuasión, el manejo del auditorio, así como una adecuada y coherente expresión oral, de cara a la consolidación de una competencia propia del Abogado egresado del Programa de Derecho de la Universidad Surcolombiana.

\section{Evaluación por Competencias}

La evaluación de las competencias definidas y adoptadas por el Programa de Derecho se realiza mediante la aplicación de estrategias evaluativas que permiten generar evidencias de su cumplimiento, en los ámbitos de conocimiento y desempeño como se indican a continuación, tras considerar que las 
mismas configuran un sentido enunciativo mas no taxativo, siendo vălidas aquellas que respeten la libertad de cátedra y que conduzcan a la concreción de los aspectos teleológicos del Programa.

\section{Competencia cognitiva disciplinar}

En tratándose de ésta competencia, el Programa reafirma su compromiso con el rigor, la dedicación y la exigencia necesaria para la formación de un buen abogado en los aspectos sustantivos, para lo cual, necesariamente se demanda de los educandos la constante actualización, indagación e investigación de los aspectos normativos propios de cada área, con el fin de lograr formación adecuada y pertinente. Ello implica brindar a los estudiantes unas sólidas bases jurídicas que garanticen un óptimo y apropiado ejercicio de la profesión, garantizando que los mismos conozcan, descubran, experimenten, transformen y produzcan conocimientos jurídicos y socio-juridicos fundamentales, que les permita resolver los problemas de juris que involucran las tendencias actuales del Derecho, de tal manera que el estudiante conozca, identifique y sepa hacer lo que conoce.

Para tales efectos se implementa una estrategia novedosa y efectiva de aprendizaje en relación con la consecución de objetivos, consistente en el estudio y evaluación de casos concretos, junto a los precedentes, la jurisprudencia, y las situaciones o casos hipotéticos que deberán ser resueltos por los estudiantes. Por ello, al finalizar cada curso el estudiante habrá construido conjuntamente con el docente una respuesta académica, de la mano de los nuevos recursos y tecnologías como implementos adecuados para el desarrollo didáctico, a travếs de la implementación de actividades como el seminario alemán, conversatorios, mesas redondas, debates, foros, talleres, el trabajo grupal, el estudio y documentación de casos o expedientes, lo que le permitirá al estudiante desarrollar aptitudes durante su proceso de formación.

\section{Competencia de adminis tración del conflicto}

El Abogado del Programa de Derecho de la Universidad Surcolombiana, debe tener la capacidad para prevenir, identificar, proponer soluciones, hacer control y seguimiento del conflicto e intervenir en él mediante su manejo integral. Esto traduce la necesidad de construir en el egresado una conciencia del papel de mediador y facilitador que tiene bajo su condición de abogado; en la capacidad para tratar de manera armónica el conflicto desde las perspectivas extrajudiciales y judiciales, con la justa y debida proporción, distancia y respeto que el mismo demanda, tras el reconocimiento pleno que su papel apunta decididamente a la construcción de un orden justo y al logro de la convivencia pacífica a través de la resolución de los conflictos. 
Con el ánimo de conseguir que et Abogado Surcolombiano intervenga como mediador, en forma individual o colectiva, con imparcialidad y empatía, propiciando el desarrollo de una cultura de paz, diálogo y debate desde la perspectiva del derecho, el respeto, la ética y el profesionalismo, mediante el reconocimiento de la otredad y la articulación de las divergencias de opinión dirigida a la proposición de soluciones equitativas y razonables, el Programa propende por la enseñanza de los aspectos judiciales y extrajudiciales, con un adecuado manejo de los elementos técnicos de la profesión durante el desarrollo de las diferentes asignaturas que lo permitan, lo que se ve reforzado con los cursos de Mecanismos Alternativos de Solución de Conflictos (MASC) y las prácticas realizadas por los estudiantes en Consultorio II (antigua pre-práctica jurídica) y en el Centro de Conciliación durante su último semestre de Consultorio.

\section{Competencia comunicativa}

Pese a que existe una competencia genérica al interior de la Universidad que evalúa esta capacidad, se considera sumamente necesaria la inclusión de la misma en forma específica al Programa de Derecho como un componente de profundización, al ser éste necesario para desarrollar la capacidad de comprender y utilizar de manera precisa el lenguaje jurídico, con el fin gue el estudiante pueda comunicar sus ideas con claridad y coherencia en todos los escenarios del ejercicio de su futura profesión.

Durante las discusiones sostenidas al interior del Comité de Currículo del Programa de Derecho, se ha logrado identificar la destacada falencia que comportan la comunidad estudiantil en la actualidad, en relación con la comprensión lectora, escritura y escucha, componentes gue exigen un nivel más elevado en la formación jurídica y ejercicio del Derecho, pues resuita constante la lectura del sistema normativo, la necesidad de comprender el lenguaje jurídico y expresarlo o comunicarlo con precisión y propiedad, incluyendo la capacidad de elaborar argumentos y alegatos jurídicos coherentes, congruentes y debidamente motivados, dentro de cualguier ámbito del ejercicio profesional.

En consecuencia y sin perder de vista la obligación del Programa de preparar a los estudiantes para la presentación de pruebas externas estandarizadas, como las Saber $\mathrm{Pro}_{r}$ se adoptan distintos mecanismos que permitan el cultivo de estas habilidades, tras la lectura de textos, la realización de escritos tipo ensayos y la implementación de preguntas abiertas en los parciales, de tal forma que pueda evaluarse la capacidad de redacción, la coherencia, la síntesis, la argumentación, la claridad de las ideas, la precisión de los términos jurídicos, así como la creación de conocimiento autónomo y crítico por parte del estudiante, lo cual se desarroliado con profundidad específica en la asigna- 
tura Consultorio Jurídico I (antigua técrica de elaboración de documentos). Esta capacidad es igualmente fomentada en forma extracurricular, durante los procesos de capacitación de los estudiantes que participan en los diferentes concursos tanto nacionales como internacionales de Derechos Humanos, los cuales requieren habilidades especiales de redacción, oralidad análisis y argumentación, pues giran en torno a la figura de un moot court.

Por su parte la labor docente es asumida como una acción conjunta entre educadores y educandos, en la que el proceso de aprendizaje es mutuo y reciproco. Es propio de esta dinámica la búsqueda y el descubrimiento común de lo novedoso, donde el futuro profesional participa plenamente al lado del maestro, va aprendiendo a aprehender, al tiempo que rompe paradigmas para dar cabida a nuevos aprendizajes sustitutivos de los anteriores con lo cual adquiere autonomía, como un insumo que más adelante resultará útil para su vida; un ejercicio del auto-aprendizaje y de auto-formación que se requiere para mantener actualidad en el campo profesional, tras cultivar los hábitos de lectura y actualización constante como una virtud profesional. De esta forma, el interés del estudiante se centra en el desarrollo de la capacidad de pensar, de resolver problemas y de afrontar exitosamente las situaciones nuevas.

Por ello la debida programación y articulación del tiempo dedicado al trabajo independiente bajo la modalidad de formación por créditos académicos, implica la planificación rigurosa y detallada de los docentes, con selección de material bibliografico, de jurisprudencia, doctrina y demás lecturas actualizadas y pertinentes seleccionadas a título de básicas, de profundización o complementarias, cuya recurrencia permita el debate, la socialización conjunta, el dialogo y la indagación multilateral, lo que además de fortalecer el proceso de formación, incentiva la habilidad lecto-escritura, la expresión oral y el adecuado manejo de estrategias comunicativas en ef estudiante que le permiten desenvolverse con seguridad en los diferentes escenarios de desarrollo.

\section{Competenciade investigación jurídica}

Resulta evidente la preeminencia de la investigación dentro del proceso de formación del Abogado Surcolombiano, desde los ámbitos curriculares y extracurriculares. Por tanto se encuentra en diferentes momentos del quehacer académico, pues además de estar presente en el desarrollo de las asignaturas de conocimiento y ser una modalidad de grado mediante la realización de una Monografía ode una investigación a través de un Semillero de Investigación, se encuentra establecida en los cursos Teoría General de la Investigación, Seminario de Investigación I y Seminario de Investigación II. 
Dada la importancia para el Programa de esta competencia, se ha fijado la estrategia de vincular preguntas de investigación durante el desarrollo de las diferentes asignaturas, las que sin pretender un rigor investigativo exhaustivo, permiten al estudiante la posibilidad de generar un espacio para la indagación, recopilación sistematización y análisis de información como una actividad dirigida a resolver problemas planteados bajo la orientación del docente, lo que permite una construcción conjunta del conocimiento.

\section{Competencia de la responsabilidad profesional}

Existe un consenso absoluto en la prioridad de. formar una nueva generación de abogados, cuyo compromiso social y profesional descanse en valores éticos, morales y deontológicos, que además permitan romper los paradigmas tradicionales de la profesión y enaltecerla. Portal razón el Programa de Derecho ha incluido dentro del componente básico el curso de Responsabilidad Profesional, preceptos que se cultivan a lo largo de la formación, pues se trata de un deber legal y constitucional, así como un compromiso con la sociedad, formar personas con un alto grado de responsabilidad social, caracterizado por su diligencia, competencia y rectitud.

\section{Abogado de audiencia}

A la par de las nuevas tendencias procedimentales en nuestro país, donde la implementación de los procesos orales se proyecta como uno de los ajustes importantes para la eficacia y celeridad de ta justicia, el Programa busca estándares que permitan el desarrollo de destrezas en las técnicas del juicio oral, la oralidad y la oratoria, la persuasión, el manejo de auditorio, una adecuada y coherente expresión oral entre otros aspectos, como un signo distintivo del Abogado Surcolombiano.

Dicha competencia es cultivada y evaluada desde los ámbitos curriculares y extracurriculares. El primero de los presupuestos cuenta con los programas de Oralidad, Oratoria, Técnicas del Juicio Oral, Procedimiento Civil, Derecho Laboral y Derecho Procesal Laboral, lo que se complementa con la implementación de estrategias didácticas como las exposiciones, talleres, o debates en general, así como el esłudio y argumentación de casos y la evaluación oral. El segundo de ellos se refiere a la preparación que el Programa realiza a los estudiantes en el marco de los concursos de Derechos Humanos en los que tradicionalmente participa.

\subsection{Componente Interdisciplinario del Programa de Derecho.}

El tema del componente interdisciplinario indudablemente fue uno de los aspectos de mayor relevancia y discusión durante el proceso de au- 
toevaluación con fines de acreditación, en donde luego de analizada la información obtenida y la revisión documental necesaria, se identificó por parte del Comité la existencia de una política institucional y de Programa en relación con la interdisciplinariedad, aunque en ciertos aspectos se encontró una confusión conceptual por parte de los estudiantes sobre el tema, así como la carencia de interés por parte del mismo estamento a la hora de acceder a escenarios del conocimiento distinto al área jurídica, tras considerarlos "relleno" o"costura".

Portal razón resulta necesario reconocer y enfatizar en la importancia de la integralidad en la formación de los futuros profesionales con el objetivo de estimular este tipo de formación, diseñando y ofreciendo cursos básicos, electivos y complementaros pertenecientes al componente humano. Por eilo como resultado del proceso, se revisó la malla curricular a fin de optimizar la ubicación de las distintas materias básicas, complementarias y electivas (tanto de formación integral como de profundización jurídica), para que su asignación responda al proceso evolutivo del estudiante durante los diferentes semestres. Además como estrategias de mejoramiento se establecieron las de definir y socializar el concepto de la interdisciplinariedad y su importancia en el proceso de enseñanza aprendizaje; delimitar las electivas por bioques, de manera tal que en los primeros semestres se ubiquen aquellas transversales de formación integral, dejando las de profundización jurídica para semestres superiores con el ánimo de posibilitar que los estudiantes profundicen en las áreas de su mayor interés; realizar una evaluación permanente de las líneas de investigación para ei planteamiento de las electivas de profundización, así como la realización de unas estadísticas y sondeo de las electivas más aceptadas.

Respecto de la estructura curricular del Programa de Derecho, podemos señalar que propone una conjunción integral entre teoría y práctica, que permite la comprensión de los fenómenos socio-jurídicos bajo dimensiones conceptuales e intelectuales coherentes. Ei currículo enlaza los contenidos del campo de la fundamentación con los niveles de formación profesional, con la intención de construir procesos interdisciplinarios que permitan la conformación de comunidades académicas en los cuales se generen espacios para compartir criterios, propiciar consensos, admitir diferencias, construir saberes con pertinencia académica en cuanto a contenidos y metodologías, concebido como un proceso de construcción permanente y colectivo, donde la investigación es asumi- 
da como un compromiso y una disposición constantes por parte de los docentes y los estudiantes con el propósito de renovar y fortalecer los procesos formativos. La revisión curricular será permanente, toda vez que los cambios que se presentan en los contextos social, político, económico y cultural, marcan cada vez nuevos senderos en el devenir histórico y jurídico del País y del Mundo.

El diseño del currículo está pensado en el análisis y búsqueda de escenarios de participación y de comprensión de fenómenos sociales, políticos y jurídicos que propicien procesos de autoformación responsable, de socialización del conocimiento y de crecimiento colectivo de todos los actores involucrados. La propuesta curricular busca generar procesos pedagógicos activos que incentiven la autonomía del estudiante, potencie sus habilidades en escenarios constructivos que apunten a incentivar las inteligencias múltiples, tal como lo señala Howard Gardner. ${ }^{46}$

En este sentido, el currículo avanza hacia el mejoramiento del programa de formación en Derecho, toda vez que la tarea fundamental de la educación es "el desarrollo de competencias intelectuales y simbólicas, en general, y de roles/perfiles intelectuales, en particular, en el contexto de cada plan de estudios ${ }^{n t 4}$; puesto que el arte de enseñar no es una ciega adopción de fórmulas y métodos, sino una clara comprensión de múltiples factores que intervienen en el proceso educativo, en donde el estudiante irá formándose en conocedor crítico de la historia, conceptos y sistemas Socio jurídicos.

\section{CONCLUSIONES}

Se propone la adopción de un Modelo Pedagógico de Indagación Sistemática, estructurado en Núcleos Temáticos Problemáticos, caracterizado por la Disciplinariedad, la Interdisciplinariedad, la Trasdisciplinariedad y la Complementariedad, desde perspectiva altemativa y neosistémica, tras la adopción de estrategias que involucren un proceso de divulgación efectivo entre el cuerpo docente y estudiantil que permita trascender lo meramente explicativo para centrarse en el logro de una verdadera asimilación por parte de los estamentos, especialmente el de maestros.

46. Gerdner distingue seis tipos de intellgencias humanas; 目 lingüística, a musical, la lógico matemälica, b espacial, a cenestésico corporal, las inteligencias personales (nntrapersonal e impersonat), que aparecen conectadas mediante desarronos simbólicos y apropiación de lenguajes especificos. En; GARDNER. Howard Estucturas de ra Mente: La feorla of las Intoligencias Mültipies. Editorial. Fondo de Cultura Econónica. México, 1994. Página 56.

47 GONZALEZ, Julian. Memorias: Seminario Taller de Aclualización y Mejoramento Curricular de los PTogramas de Formación an Comunicación Social. Sankiago de Cali. 1996 
Este modelo de complementariedad, está basado en la formación por competencias, con el fin de logr ar una proceso de aprendizaje integral, flexible, interdisciplinario y transdisciplinario, en donde se interrelacioné lo disciplinar, lo fáctico, el desarrollo de habilidades y el desarrollo humano. Igualmente, integra estrategias didácticas innovadoras y renovadoras del proceso de formación, dentro de una construcción conjunta de conocimiento, identificando en los estudiantes uno interlocutores válidos de cara a las nuevas herramientas digitales.

Como consecuencia de lo anterior, se puede observarse de acuerdo con la revisión documental analizada, que a nivel institucional existen problemas en cuanto a la definición de un modelo por competencias, la carencia de una definición epistemológica de los aspectos curriculares y pedagógicos y la comprensión de la flexibilidad, la interdisciplinariedad y la transdisciplinariedad.

\section{Referentes Bibliográficos}

MODERNIZACIÓN CURRICULAR DE LA UNIVERSIDAD SURCOLOMBIANA Una Experiencia de Integración e Interdisciplinariedad; López Jiménez Nelson Ernesto, Puentes de Velázguez Ana Victoria.

La Enseñanza del Derecho en el Siglo XXI Una guía práctica sobre el Grado en Derecho, Edición 2009, Editorial Aranzad. España. De los Doctores JAIME RODRIGUEZ ARANA MUNOZZ, RAFAEL PALOMINO LOZANO (Directores) MARIA GOÑI RODRÍGUEZ DE AÑMEDIA y SILVIA MESEGUERVELASCO (Coordinadores).

La enseñanza del Derecho en Colombia 1886-1930" En: Colombia 2010. Editorial Universitaria - Universidad de Nariño de la Doctora ISABEL GOYES MORENO.

LA DE-CONSTRUCCIÓN CURRICULAR; LÓPEZ JIMÉNEZ Nelson Ernesto. Colección Seminarium Magisterio. 2001.

SALCEDO SÁNCHEZ, Jaime y RAMÍREZ PERDOMO, Andrea: Propuesta Antropogogica para la Facultad de Salud de la Universidad Surcolombiana, Neiva 2009.

TAPIERO VÁSQUEZ, Elías y Otro. Currículo Neosistémico y Desarrollo Institucional Integrado. Universidad de la Amazinía. Florencia 2010.

ELSI OLAYA ESTEFAN M.D., -Didáctica Para La Enseñanza De Las Ciencias De La Salud.

EISENMAN, Charles. Objetivos y naturaleza de la enseñanza del derecho.

LOPEZ, Diego. Teoría Impura del Derecho. Editorial Temis. Bogotá, 2003. 
AMAYA DE OCHOA., Graciela. La Escuela, El Maestro y su Formación. Documento Presentado a la Misión de Ciencia, Educación y Desarrollo. Santafé de Bogotá, D. C. 1.994.

ACOFADE y Universidad Militar Nueva Granada. Revista La educación y el derecho, la formación del jurista en el siglo XXI - I y II.

FLOREZ, Rafael. Hacia una Pedagogía del Conocimiento. Mc. Graw Hill. Santafé de Bogotá, D.C. 1.994.

BÖHMER MARTIN F. Compilador. La enseñanza del derecho y el ejercicio de la abogacía. Biblioteca Yale de Estudios Jurídicos. Editorial Gedisa 1999.

GARDNER, Howard. Estructuras de la Mente: La teoria de las Inteligencias Múltiples. Editorial. Fondo de Cultura Económica. México, 1994. Página 5ó.

GONZÁLEZ, Julián. Memorias: Seminario Taller de Actualización y Mejoramiento Curricular de los Programas de Formación en Comunicación Social. Santiago de Cali. 199ó.

PARAMO, Pablo. Diseño Ambiental del Entorno Escolar. Revista Educación y Cultura No. 38. CEID - FECODE, agosto de 1.995.

PORLAN, Rafael. Constructivismo y Escuela. Diada Editorial. S.L. Sevilla, 1.995.

VASCO, Eloisa. Maestros, Alumnosy Saberes. Cooperativa Editorial Magisterio. Santafé de Bogotá, D.C. 1.994.

ZULUAGA, Olga Lucia. Pedagogía e Historia. Ediciones Foro Nacional por Colombia Santafé de Bogotá, D. C. 1.987.

GUERRA, Yolanda. Métodos en Investigación ¿Todos los caminos conducen a Roma?

DE GREGORI, Waldemar. Cibernética Social. Administración Sistemática. Edición ASIC-PRO.

LEY GENERAL DE LA EDUCACION. Decretos Reglamentarios, Plan Decenal de Educación Compilación, Corporación. Tercer Milenio. 